\title{
Research on Design of Flexible Fence in Racecourse
}

\author{
Hanhua Zhang a , Shuang Zhang ${ }^{\text {b, * }}$ \\ International School of Equestrian, Wuhan Business University, Wuhan 430056, China \\ azhangshuangyq@163.com, btiredhiker@hotmail.com
}

Keywords: racecourse, flexible fence, safety.

\begin{abstract}
This paper has carried out field investigation and research on the fences used in the domestic and foreign racecourse through literature method, expert interview method and field investigation method. The results show that the racecourse fence structures used on the market are mainly wood, steel pipe and PVC plastic material, under special circumstances, theses fence structures are easy to cause harm to the horse and jockey in motion. For this reason, this research designs an flexible fence which is different from the traditional racecourse fence, this kind of flexible fence can effectively reduce the wounded risk of horse and jockey, improve the security of horse racing, equestrian sports. This research is of great significance to improve the safety of equestrian sports and ensure the safety of equestrian.
\end{abstract}

\section{Introduction}

The racecourse fence is a kind of isolation facility which is used to ensure the safety of horses, riders and spectators. The racecourse fence mainly delimits limited the sports areas for the horse, and achieve the purpose of isolating the audience. At present, the fence structure materials used at home and abroad are mainly wood, steel and hard plastic. All parts of wood structure fence are made of wood; steel structure fence is generally adopted by steel pipe; PVC hard plastic is used in the general board area of plastic fence, and steel pipe is adopted in the connection part. Because the support connection parts of fence on the market all adopt hard structure to connect at present, which cause fence does not have flexibility in use process, it is very easy to scratch jockey's leg and horse skin in the sports event. Special circumstances such as falling off the horse; and it can cause the whole body of riders to crash into the fence, which can easily lead to riders' internal injuries, fractures and even death. Considering the present condition of the racecourse fence and importance of fence to ensure the safety of riders and horses, this research design a flexible fence, it can play a limit role in the horses' movement area, and can protect the safety of horses and riders and the audience.

\section{Introduction of Traditional Fence}

\subsection{Wooden Fence}

Wooden fence is a type of fence that buries wood directly to the ground and fixes. Wooden fence is used by wood and the wood is usually cut into pieces or squares for installation. Wooden fence is cheap on the one hand, on the other hand, the wood are processed by shape and pattern and other decorative effects according to the need, so the wood type of fences are commonly used in some small and medium-sized equestrian club. But the fastness of the wooden fence is poor, it most easily cause person, horse safety accident. The common wooden fence of the racecourse is shown in Figure.1. 


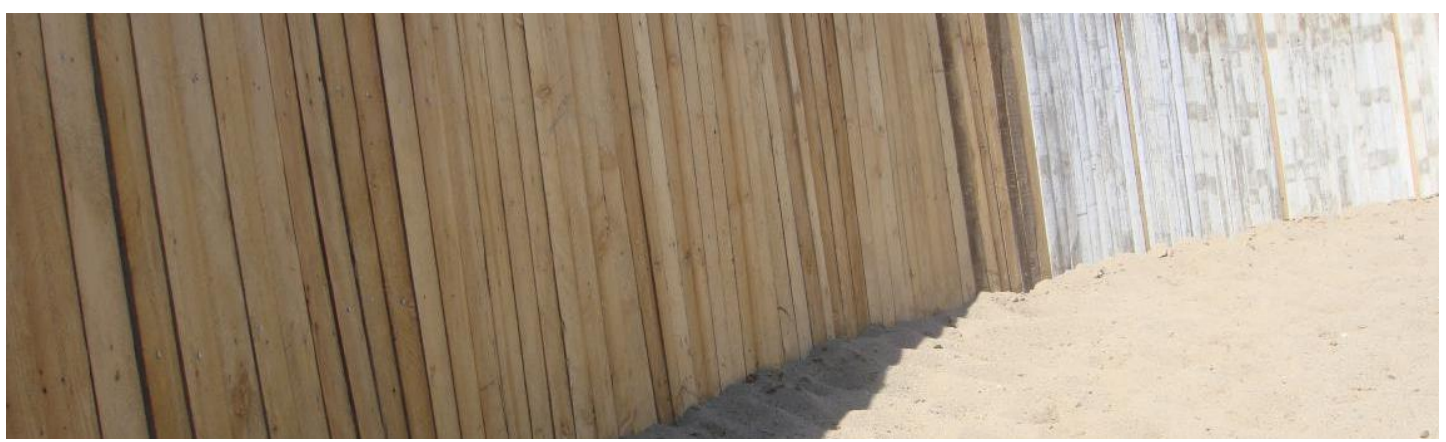

Figure.1 wooden type fence

\subsection{Steel Fence}

Steel fences are hard and durable. Steel pipe is generally used for construction. Steel fences are commonly seen in small and medium-sized equestrian clubs. Steel fence is easy to cause the riders' leg to be scraped in the process of riding horses, because hardness of steel fence is strong, When falling off the horse in the riding process, it is easy to cause the rider impacts on the fence, and cause fracture, etc. The steel type fence is shown in Figure.2.

\subsection{PVC fence}

PVC fence is a kind of fence material widely used in the middle and high-end equestrian club and racecourse. PVC fence has the advantages of beautiful color, bright, smooth and bright surface, high strength and long service life. At present, there are two types of connecting parts of PVC fence in various racecourses: for the equestrian fields with small area and small amount of fence, PVC columns are generally used to connect the ground. And for the racecourses with larger usage amount and strong support strength of the joint, the steel pipe structure is used to connect the ground. The two types of connection ways all are hard connections, and it is easy to cause injuries in special cases. PVC type fence is shown in Figure.3.

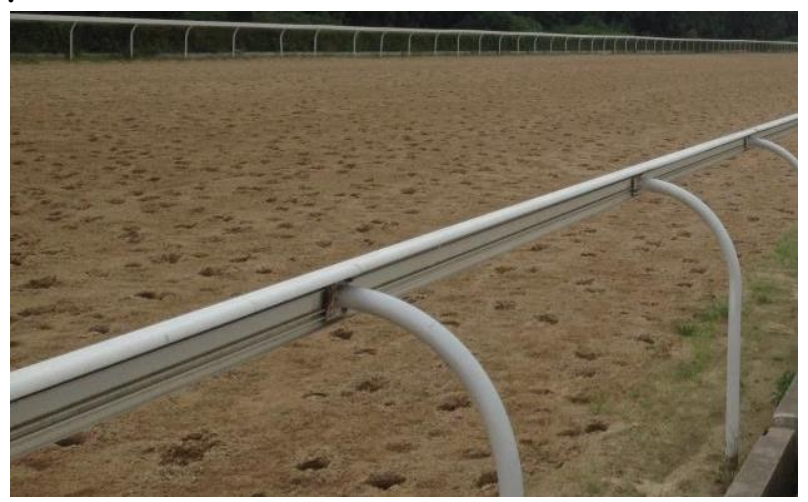

Figure.3: PVC type fence.

\section{Advantages and Disadvantages of Traditional Fences.}

Wooden fence is cheap, easy to install and other features. However, the wooden fence is prone to some disadvantages such as moth, decay and fire. Among them, the decay under natural conditions is the most difficult problem to solve. In addition, the installation of the wooden fence need to use the nails and other hardware accessories, if this processing of fittings is improper and after using for a long time, it will appear naked and rusty phenomenon, which will do great harm to the safety of the horse and rider.

Steel fence is usually installed by welding, the biggest problem of steel fences is that it will oxidize and rust when used too long. Steel fence generally adopts steel pipe as the basic material, defects of steel pipe is smaller contact nep, when horse hit the fence, it is very easy to cause the riders' leg and horses' leg skin bruise and so on. Due to the hardness of the steel fence, the fracture and internal injuries are most likely to occur when the rider fall off the horse.

The PVC fence is beautiful and light, it is the most common kind of fence structure on the market at present. The connecting pieces of PVC fence adopt plastic or steel pipe material according to the size 
of usage amount. Because fence usually need to bend certain angle inward, when connecting pieces use plastic material, due to excessive bearing of connecting pieces and result in connecting piece distort., affect beauty and use, if do not use bending angle, security problem will appear again, therefore, plastic connecting pieces are commonly used in equestrian field; in the racecourse, because of large usage amount of fence, in order to solve the problem of safety and bearing, the connecting pieces usually adopt steel pipe structure. Steel pipe connections will make the intensity of fence stronger, lack elasticity and cushion reduce, which will pose a threat to the safety of the rider and the horse.

\section{Flexible Fence Design}

\subsection{Connecting Piece of Flexible Fence.}

The structure of the fence is divided into two parts: the connecting piece and the breast board, the key part of the flexible fence is the connecting piece. The traditional connecting pieces are mostly steel pipe structure, which is directly buried in the ground; this treatment is relatively simple and strong. The connecting pieces of flexible fence are divided into two parts: the steel pipe structure which connects the breast board and the elastic buried device which handles the impact force. The connection parts of steel pipe and breast boar can be connected by stationary type, and the structure is relatively simple. Elastic ground device is the most important parts to solve the flexible fence, this part is elastic, when fence on impact, impact force is passed to the buffer device through steel pipe, the buffer device will offset impact by reacting force and displacement, so as to reduce damage. The pattern diagram of connecting piece of flexible fence is shown in Figure.4.

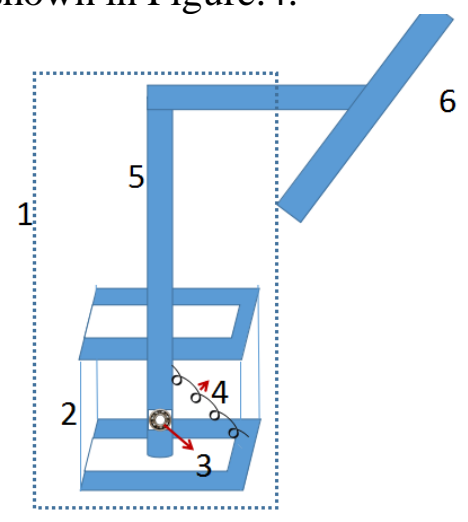

1. connection piece, 2. buried solid equipment, 3. bearing, 4. spring, 5. steel pipe, 6. breast board

Figure. 4 connecting piece of flexible fence

The working principles of connecting piece of flexible fence are as follows: elastic underground device needs to be buried deep under the ground, Because the steel pipe needs displacement space in the elastic underground device., therefore, the top of the elastic underground device needs to be exposed $10 \mathrm{~cm}$ above the surface, elastic underground devices are buried deeply, whose main effect is to strengthen the whole of fence structure. The interior of elastic underground device is hollow structure, which can provide ample space for displacement of the steel pipe, the bottom of the steel pipe adopt movable part of bearing connection, when on impact the steel pipe can be rotated around the bearing, above the bearing connection uses the spring structure to connect to the bottom of the underground device, the spring structure are able to buffer the impact and after the impact end, and return to the original position under the original tension of the spring.

\subsection{Breast Board Connection of Flexible Fence.}

Because connecting piece of flexible fence belong to elastic structure, after horse hitting the fence, it need several breast boards of fence can cause displacement of the corresponding distance, therefore, the breast board can't make it integral, only take the section form design. The specific pattern is shown in Figure.5. 


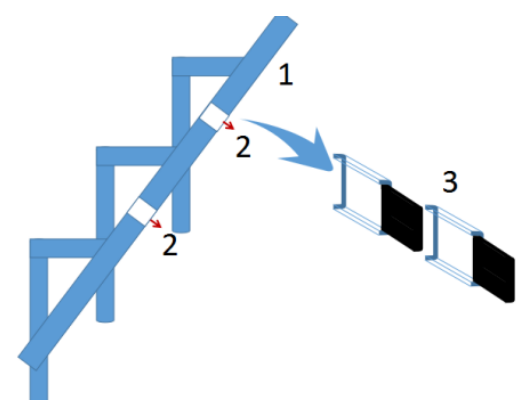

1. breast board of flexible fence; 2. connection of breast board; 3 . details of connection part of breast board

Figure. 5 breast board connection of flexible fence

The breast board of flexible fence need each column set breast board, when the horse hits the board, it can produce a good displacement, if breast board is set to the overall form, $i$ then horse hitting the board, there is not enough displacement to cushion the great impact. Moreover, The placement direction of the breast board should be determined according to running direction of the horse, the plug at the junction should be opposite to the horse's running direction, the setting of this direction can make all the columns become a whole, it is beneficial for all the elastic underground parts to participate in the buffer work.

\subsection{Working Principle of flexible Fence.}

When horses impact flexible fence, flexible fence can produce proper displacement under the action of force, at this time, the impact force of horses will be buffered, after the impact force ends, the fence will be restored to its original state under the tension of the spring. In addition, the tension of the spring inside the underground part does not need to be too large, as long as there are forces on the fence, it can produce displacement. Though impact force when horses in the running process is very big, the board connection structure in the fence will connect all the individual breast board and the elastic underground piece, and form an organic whole, thus buffering impact force at the same time. The working principle of the flexible fence is shown in Figure.6.

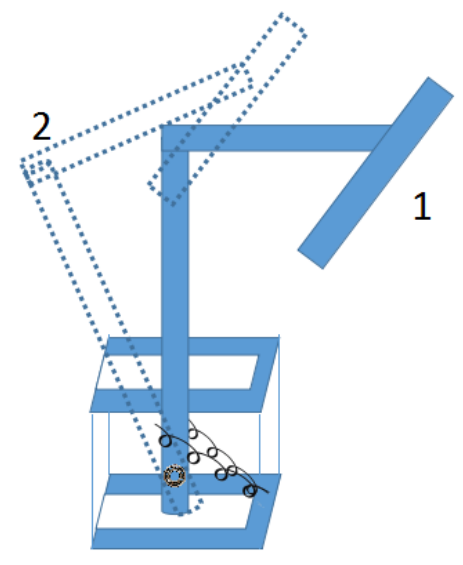

1. Initial state of fence; 2 . displacement state after fence on impact.

Figure 6: working principle diagram of flexible fence.

\section{Conclusions and Suggestions.}

The fence structure currently adopted on the market are mostly wooden structure, steel structure and PVC plastic structure, connection firmness, hardness and without cushioning horses impact force exist in these fences. Under special circumstances, it is easy to cause a certain degree of injury or even life danger to horses and riders.

The design of flexible fence can effectively reduce the occurrence probability of injury accidents caused by horse impact fence under the special circumstances, which is a relatively scientific and reasonable fence structure. The connecting pieces of flexible guardfence adopt steel structure, and the breast board adopts PVC material, which has the characteristics of beauty, firmness and durability. 
The main functional parts of the flexible fence are the elastic underground parts, this part is slightly larger and installation is more complicated, which requires a large amount of work. Therefore, in order to save cost, we should deal with the racecourse foundation; meanwhile the elastic underground pieces of the flexible fence should be placed together for disposal.

Bearing and spring are used in elastic underground piece structure of flexible fence, and the disadvantages of these devices are oxidization and rusting, therefore, we should pay attention to waterproof in use and maintain timely, and improve the service life of the fence.

\section{References}

[1] Lei Zhengbao. Vigorously Develop Research on the New Anti-Collision Mechanism of Semi-rigid Fence [J], Journal of Vibration and Shock. 2002. 21:1-6.

[2] Wang Yan, Zeng Xiantang. Summary and Discussion about Several Typical Guardfence Design Problems and Solution of High Grade Highways[J]. Highway, 2013(1): 269-274.

[3] Lei Zhengbao, Yang Zhao. Study on the Passenger Safety during the Impact Process of Automobile against Fence [J]. Journal of Vibration and Shock, 2006, 25(2):5-11.

[4] Yan Shuming, Bai Shufeng, Yu Haixia. Analysis and Resolving Method of Accidents At Median Barrier Gate [J]. Highway, 2010(1): 196-201.

[5]Fu Zhipeng, Wu Zhiyong, Jing Lin. Research on W-beam Guardfence Height of Expressway Media[J]. Journal of Highway and Transportation Research and Development, 2010, 27 (9):143-148. (in Chinese).

[6] Yang Shaowei, Pan Binghong, Wu Huajin, et.al. Median Type And Safety On Freeway[J]. China Journal of Highway and Transport, 2006, 19 (6):39-44.(in Chinese)

[7] Yan Shuming. Feasibility Analysis of Barrier Safety Evaluation with Finite Element Simulation Method[J]. Journal of Vibration and Shock, 2011, 30(1):152-156.

[8] Yan Shuming. Feasibility of Safety Performance of Fence with Finite Element Simulation Method Evaluation[J], Journal of Vibration and Shock, 2011 (1). 